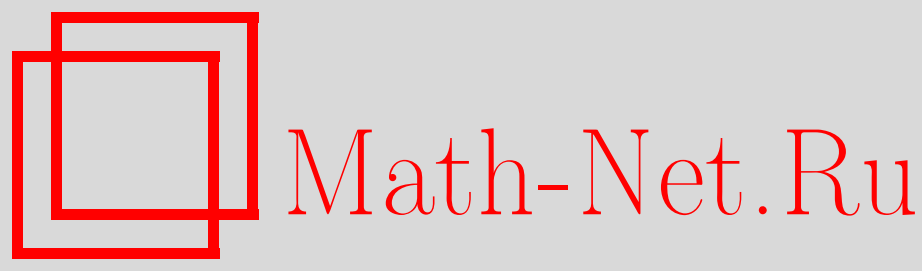

Г. Ф. Хельминк, А. Г. Хельминк, Е. А. Панасенко, Интегрируемые деформации в алгебре псевдодифференциальных операторов с точки зрения алгебраической теории Ли, ТМФ, 2013, том 174, номер 1, 154-176

DOI: https://doi.org/10.4213/tmf8362

Использование Общероссийского математического портала Math-Net.Ru подразумевает, что вы прочитали и согласны с пользовательским соглашением http://www.mathnet.ru/rus/agreement

Параметры загрузки:

IP : 3.80 .253 .173

26 апреля 2023 г., 06:48:37

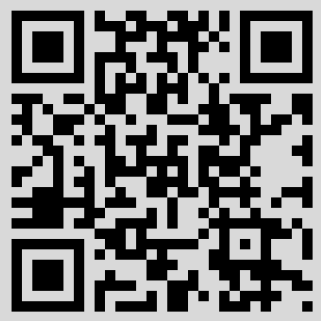




\title{
ИНТЕГРИРУЕМЫЕ ДЕФОРМАЦИИ В АЛГЕБРЕ ПСЕВДОДИФФЕРЕНЦИАЛЬНЫХ ОПЕРАТОРОВ С ТОЧКИ ЗРЕНИЯ АЛГЕБРАИЧЕСКОЙ ТЕОРИИ ЛИ
}

\begin{abstract}
Представлена (двумя различными способами) алгебра псевдодифференциальных операторов в виде прямой суммы двух подалгебр Ли и деформировано множество коммутирующих элементов одной из подалгебр в направлении другой компоненты. Эволюция деформируемых элементов приводит к двум совместным системам уравнений Лакса, которые обе имеют минимальную реализацию. Показано, что такая форма Лакса эквивалентна множеству отношений нулевой кривизны. Приводятся линеаризации указанных систем, которые являются ключевым подходом к построению решений.
\end{abstract}

Ключевые слова: интегрируемые деформации, псевдодифференциальные операторы, уравнения Лакса, иерархия Кадомцева-Петвиашвили, отношения нулевой кривизны, линеаризации.

DOI: $10.4213 / \operatorname{tmf} 8362$

\section{1. ВВЕДЕНИЕ}

В настоящей работе изучаются алгебраические аспекты двух совместных систем уравнений Лакса для псевдодифференциальных операторов. Обе системы связаны с разложением этой алгебры Ли как векторного пространства в прямую сумму двух подалгебр Ли и деформацией базисного множества коммутирующих элементов одной компоненты разложения в направлении другой компоненты. Одно из представленных разложений приводит к иерархии Кадомцева-Петвиашвили (КП), другое к строгой иерархии КП. Как показано в работе [1], обе системы появляются естественным образом в двумерной иерархии Тоды. Мы предложим минимальную реализацию обеих систем уравнений Лакса и покажем ниже в теореме 2 , что подобная форма Лакса в обоих случаях эквивалентна системе уравнений нулевой кривизны. Последнее указывает на существование системы совместных линейных уравнений.

${ }^{*}$ Korteweg-de Vries Institute, University of Amsterdam, Amsterdam, The Netherlands. E-mail: g.f.helminck@uva.nl

${ }^{\dagger}$ North Carolina State University, Raleigh, USA. E-mail: loek@math.ncsu.edu

‡Тамбовский государственный университет им. Г. Р. Державина, Тамбов, Россия. E-mail: panlena_t@mail.ru 
В заключение будет предложено описание этих линеаризаций, дающих алгебраическую постановку задачи построения решений из бесконечномерных многообразий (см. работы [2], [3]).

Порядок изложения следующий. В разделе 2 представлены аспекты алгебраической теории Ли совместных систем уравнений Лакса в пространстве конечномерных матриц. Затем представляется алгебра псевдодифференциальных операторов, ее наиболее значимые свойства и разложения. Раздел 4 посвящен описанию систем уравнений Лакса в этой алгебре. В разделе 5 показано, что существует реализация обеих систем с минимальным числом соотношений между коэффициентами деформированных операторов. В разделе 6 доказывается, что форма Лакса уравнений эквивалентна форме нулевой кривизны. Линеаризации описаны в заключительной части работы.

\section{2. СОВМЕСТНЫЕ УРАВНЕНИЯ ЛАКСА ДЛЯ МАТРИЦ}

Рассмотрим дифференцируемое отображение $t \mapsto g(t), t \in \mathbb{R}$, действующее во множестве обратимых $(n \times n)$-матриц. Каждую $(n \times n)$-матрицу $V$ можно деформировать, сопрягая ее с заданным отображением, что приводит к потоку

$$
t \mapsto L(t):=g(t)^{-1} V g(t) .
$$

Обозначим $M(t):=(d g(t) / d t) g(t)^{-1}=\dot{g}(t) g(t)^{-1}$. Тогда прямые вычисления показывают, что эволюция деформации $L$ матрицы $V$ может быть задана в виде

$$
\dot{L}:=\frac{d L}{d t}=L M-M L=[L, M] .
$$

Уравнение (2) является примером так называемого уравнения Лакса. Многие системы в механике имеют такую форму (см., например, работу [4]), прототипом которой является конечная открытая решетка Тоды (для сравнения см. работу [5]). Их связь с теорией представлений подробно исследована в работе [6].

Следующим шагом является изучение систем совместных уравнений вида (2). Обыкновенные производные в этом случае заменяются на частные производные. Мы вкратце напомним (сначала для $(n \times n)$-матриц), как с точки зрения алгебраической теории Ли получаются совместные системы уравнений Лакса. Это послужит основой для изучения бесконечномерного случая.

Мы начнем с вещественной или комплексной алгебры Ли $\mathfrak{g}(n \times n)$-матриц и соответствующей ей матричной группы Ли $G$. Будем предполагать, прежде всего, что алгебра Ли $\mathfrak{g}$ как векторное пространство представима в виде прямой суммы

$$
\mathfrak{g}=\mathfrak{g}_{1} \oplus \mathfrak{g}_{2}
$$

ее подалгебр Ли $\mathfrak{g}_{1}$ и $\mathfrak{g}_{2} ;$ соответствующие проекции будем обозначать $\pi_{i}: \mathfrak{g} \rightarrow \mathfrak{g}_{i}$. Следующее необходимое условие заключается в том, что каждая из $\mathfrak{g}_{i}, i=1,2$, есть алгебра Ли подгруппы Ли $G_{i}$ группы $G$. Оно не представляет сложности в конечномерной ситуации, но выполнение его в бесконечномерном случае может оказаться проблематичным.

Далее, мы должны предположить, что разложение в прямую сумму имеет свой эквивалент на групповом уровне, а именно отображение $\left(g_{1}, g_{2}\right) \mapsto g_{1} g_{2}$ является диффеоморфизмом из $G_{1} \times G_{2}$ в $G$. Это свойство может не иметь места как в конечномерном, так и в бесконечномерном случае. 
Системы уравнений Лакса, которые мы хотим исследовать, связаны с коммутирующими потоками на $G_{2}$. Рассмотрим семейство $\left\{F_{i} \mid F_{i} \in \mathfrak{g}_{2}, 1 \leqslant i \leqslant m\right\}$ матриц, которые, прежде всего, линейно независимы и, кроме того, коммутируют между собой:

$$
\left[F_{i_{1}}, F_{i_{2}}\right]=0
$$

для любых $i_{1}$ и $i_{2} \in\{1, \ldots, m\}$. Тогда соответствующие коммутирующие потоки суть

$$
\gamma(t)=\gamma\left(t_{1}, \ldots, t_{m}\right)=e^{\sum_{i=1}^{m} t_{i} F_{i}} .
$$

Теперь деформируем образующие коммутирующих потоков в $\mathfrak{g}_{2}$ в направлении $G_{1}$ и рассмотрим эволюцию этих деформаций. Для этого возьмем произвольный $g \in G$ и коммутирующие потоки $\gamma$, определенные формулой (5). В силу единственности разложения $G=G_{1} G_{2}$ найдутся такие элементы $g_{1} \in G_{1}$ и $g_{2} \in G_{2}$, что

$$
\gamma(t) g \gamma(t)^{-1}=g_{1}(t)^{-1} g_{2}(t)
$$

Далее используем компоненту $G_{1}$, чтобы деформировать направления в $\mathfrak{g}_{2}$, а именно определим многомерные потоки $\mathcal{F}_{i}$ в $\mathfrak{g}$ равенствами

$$
\mathcal{F}_{i}:=g_{1} F_{i} g_{1}^{-1}, \quad 1 \leqslant i \leqslant m .
$$

Такая деформация сохраняет коммутативность:

$$
\left[\mathcal{F}_{i_{1}}, \mathcal{F}_{i_{2}}\right]=0
$$

Эволюция $\left\{\mathcal{F}_{i}\right\}$ относительно параметров коммутирующих потоков задается уравнениями Лакса. Точнее, имеет место следующая теорема.

Теорема 1. В используемых обозначениях дебормации $\left\{\mathcal{F}_{i}\right\}$ исходных коммутирующих направлений удовлетворяют равенствам

$$
\frac{\partial}{\partial t_{i_{1}}}\left(\mathcal{F}_{i_{2}}\right)=\left[\mathcal{F}_{i_{2}}, \pi_{1}\left(\mathcal{F}_{i_{1}}\right)\right]=\left[\pi_{2}\left(\mathcal{F}_{i_{1}}\right), \mathcal{F}_{i_{2}}\right] .
$$

ДоказАтельство. Отметим, что второе из равенств (9) является следствием того факта, что деформации $\left\{\mathcal{F}_{i}\right\}$ коммутируют, а также тождества

$$
\pi_{1}\left(\mathcal{F}_{i_{1}}\right)=\mathcal{F}_{i_{1}}-\pi_{2}\left(\mathcal{F}_{i_{1}}\right)
$$

Так как все $\left\{\mathcal{F}_{i}\right\}$ получены сопряжением постоянной матрицы матрицей, зависящей от параметров $\left\{t_{i}\right\}$, можно непосредственно проверить, что

$$
\frac{\partial}{\partial t_{i_{1}}}\left(\mathcal{F}_{i_{2}}\right)=\left[\frac{\partial}{\partial t_{i_{1}}}\left(g_{1}\right) g_{1}^{-1}, \mathcal{F}_{i_{2}}\right] .
$$

Таким образом, достаточно показать, что коммутаторы в правых частях уравнений (10) совпадают с заявленными в теореме. Для этого продифференцируем выражения $g_{1} \gamma g$ и $g_{2} \gamma$ по $t_{i_{1}}$. Получим

$$
\begin{aligned}
\frac{\partial}{\partial t_{i_{1}}}\left(g_{1}\right) \gamma g+g_{1} F_{i_{1}} \gamma g & =\left(\frac{\partial}{\partial t_{i_{1}}}\left(g_{1}\right) g_{1}^{-1}+\mathcal{F}_{i_{1}}\right) g_{1} \gamma g \\
\frac{\partial}{\partial t_{i_{1}}}\left(g_{2}\right)+g_{2} F_{i_{1}} \gamma & =\left(\frac{\partial}{\partial t_{i_{1}}}\left(g_{2}\right) g_{2}^{-1}+g_{2} F_{i_{1}} g_{2}^{-1}\right) g_{2} \gamma
\end{aligned}
$$


и в силу равенства $g_{1} \gamma g=g_{2} \gamma$ имеем

$$
\frac{\partial}{\partial t_{i_{1}}}\left(g_{1}\right) g_{1}^{-1}+\mathcal{F}_{i_{1}}=\frac{\partial}{\partial t_{i_{1}}}\left(g_{2}\right) g_{2}^{-1}+g_{2} F_{i_{1}} g_{2}^{-1} .
$$

Поскольку $F_{i_{1}} \in \mathfrak{g}_{2}$, то $g_{2} F_{i_{1}} g_{2}^{-1} \in \mathfrak{g}_{2}$. Будучи касательным вектором потока в $G_{2}$, перенесенным в начало координат, слагаемое $\left(\partial g_{2} / \partial t_{i_{1}}\right) g_{2}^{-1}$ принадлежит $\mathfrak{g}_{2}$, и поэтому вся правая часть равенства (11) лежит в $\mathfrak{g}_{2}$. Аналогичные рассуждения показывают, что $\left(\partial g_{1} / \partial t_{i_{1}}\right) g_{1}^{-1}$ принадлежит $\mathfrak{g}_{1}$, а слагаемое $\mathcal{F}_{i_{1}}$ в равенстве $(11)$ как $G$-сопряжение матрицы в $\mathfrak{g}$ принадлежит $\mathfrak{g}$. Таким образом, $\mathfrak{g}_{1}$-компонента в левой части равна нулю. Отсюда получаем

$$
\frac{\partial}{\partial t_{i_{1}}}\left(g_{1}\right) g_{1}^{-1}=-\pi_{1}\left(\mathcal{F}_{i_{1}}\right)
$$

что приводит к уравнениям Лакса (9). Теорема доказана.

Предположим теперь, что вместо вещественных или комплексных $(n \times n)$-матриц у нас есть бесконечномерная алгебра Ли, состоящая из интегральных и дифференциальных операторов. Совместные системы уравнений Лакса, которые мы рассмотрим для этой алгебры, являются обобщениями формы Лакса уравнения Кортевегаде Фриза $(К д \Phi)$. Напомним, что это уравнение описывает изменение во времени высоты волны $u(x, t)$, распространяемой на поверхности воды в узком канале:

$$
u_{t}:=\frac{\partial u}{\partial t}=\frac{1}{4} \frac{\partial^{3} u}{\partial x^{3}}+\frac{3}{2} u \frac{\partial u}{\partial x}=\frac{1}{4} u_{x x x}+\frac{3}{2} u u_{x} .
$$

Уравнение КдФ может быть записано как уравнение Лакса для дифференциальных операторов относительно $\partial=\partial / \partial x$ с коэффициентами из кольца $R$ дифференцируемых функций переменных $x$ и $t$.

Для любых $a \in R$ и $m \in \mathbb{N}$ пусть $a \partial^{m}$ есть эндоморфизм $R$, представляющий собой дифференцирование $m$ раз по переменной $x$, а затем умножение на $a \in R$. Конечные суммы таких операторов называются дифференциальными операторами относительно $\partial$ с коэффициентами из $R$. Они образуют алгебру, которую мы будем обозначать $R[\partial]$.

Уравнение КдФ может быть записано теперь как равенство операторов из $R[\partial]$. Чтобы убедиться в этом, сопоставим каждой функции $v \in R$ дифференциальные операторы

$$
\mathcal{L}_{2}=\partial^{2}+v, \quad P_{3}=\partial^{3}+\frac{3}{2} v \partial+\frac{3}{4} \partial(v) .
$$

Так как $P_{3}$ и $\mathcal{L}_{2}$ есть эндоморфизмы кольца $R$, мы можем говорить о коммутаторе $\left[P_{3}, \mathcal{L}_{2}\right]$. Прямые вычисления показывают, что

$$
\left[P_{3}, \mathcal{L}_{2}\right]=\frac{1}{4} v_{x x x}+\frac{3}{2} v \partial(v) .
$$

Другими словами, оператор $\left[P_{3}, \mathcal{L}_{2}\right]$ является оператором нулевого порядка относительно $\partial$. Далее будем рассматривать $t \mapsto \partial^{2}+v(x, t)$ как поток операторов Шредингера, а $\partial_{t}:=\partial / \partial t$ - как оператор, действующий покоэффициентно на элементы $R[\partial]$, одним из которых является $\mathcal{L}_{2}$. Отметим, что утверждение " $v$ есть решение уравнения КдФ" равносильно следующему уравнению Лакса:

$$
\frac{\partial}{\partial t}\left(\mathcal{L}_{2}\right)=0 \cdot \frac{\partial^{2}}{\partial x^{2}}+2 \frac{\partial v}{\partial t}=\left[P_{3}, \mathcal{L}_{2}\right] .
$$


Первым уравнение КдФ в таком виде записал Лакс [7], что объясняет название форма Лакса уравнения КдФ для (14). Чтобы понять, как появилось уравнение (14), нужно перейти к соответствующему расширению $R[\partial]$ - алгебре псевдодифференциальных операторов относительно $\partial$. Можно доказать, что в этой алгебре существует квадратный корень $L=\left(\mathcal{L}_{2}\right)^{1 / 2}$ из $\mathcal{L}_{2}$ вида

$$
L=\partial+\sum_{j=1}^{\infty} \ell_{j+1} \partial^{-j}
$$

Тогда $P_{3}$ есть компонента алгебры $R[\partial]$ третьей степени $L$, и уравнение

$$
\left[P_{3}, \mathcal{L}_{2}\right]=\left[\mathcal{L}_{2}, L^{3}-P_{3}\right]
$$

объясняет, почему $\left[P_{3}, \mathcal{L}_{2}\right]$ является оператором нулевого порядка относительно $\partial$. Подобная алгебра псевдодифференциальных операторов является также подходящей средой для изучения совместных систем уравнений Лакса вида (14) и составляет тему следующего раздела.

\section{3. КОЛЬЦО $R\left[\xi, \xi^{-1}\right)$ ПСЕВДОДИФФЕРЕНЦИАЛЬНЫХ ОПЕРАТОРОВ}

Целью настоящего раздела является описание расширения множества дифференциальных операторов, которое объясняет форму Лакса таких уравнений, как уравнение КдФ и его обобщения. Чтобы подчеркнуть алгебраический характер наших рассуждений, а также для рассмотрения на равных вещественных и комплексных решений, мы начнем абстрактно, с алгебры $R$ над полем $k$ нулевой характеристики, которая будет служить источником решений интересующих нас нелинейных уравнений. Далее, в качестве замены операторам $\partial / \partial x$ в КдФ-постановке предположим, что на $R$ задана линейная относительно $k$ операция дифференцирования $\partial: R \mapsto R$. В силу $k$-линейности $\partial$ поле $k$ лежит в кольце констант

$$
R_{\mathrm{const}}=\{r \in R \mid \partial(r)=0\}
$$

внутри $R$. Приведенные ниже примеры являются прототипами для такой исходной постановки.

Пример 1. Пусть $R$ есть кольцо $k\left[t_{i}\right]$ многочленов переменных $\left\{t_{i} \mid i \in I\right\}$ с коэффициентами из $k$ или кольцо формальных степеннь́х рядов $k\left[\left[t_{i}\right]\right]$ тех же переменных, и пусть $\partial$ есть частная производная по одной из переменных, например $t_{i_{0}}$.

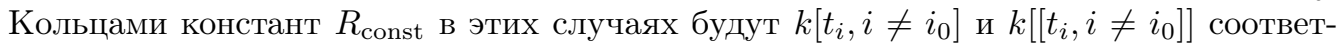
ственно.

Если $R$ и $\partial$ заданы, то можно построить дифференциальные операторы относительно $\partial$ с коэффициентами из $R$. Они образуют семейство $R[\partial] k$-линейных эндоморфизмов $R$ вида $\sum_{i=0}^{n} a_{i} \partial^{i}, a_{i} \in R$, т. е. отображений

$$
r \rightarrow \sum_{i=0}^{n} a_{i} \partial^{i}(r)
$$

действующих из $R$ в $R$. 
Правило

$$
\partial^{m} \circ r_{1}=\sum_{i=0}^{m}\left(\begin{array}{c}
m \\
i
\end{array}\right) \partial^{i}\left(r_{1}\right) \partial^{m-i}
$$

определяет операцию композиции двух таких эндоморфизмов $\sum_{i} a_{i} \partial^{i}$ и $\sum_{j} b_{j} \partial^{j}$. Результатом является

$$
\sum_{i, j} \sum_{k=0}^{i}\left(\begin{array}{l}
i \\
k
\end{array}\right) a_{i} \partial^{k}\left(b_{j}\right) \partial^{j+i-k},
$$

т. е. снова элемент $R[\partial]$. Может оказаться, что степени $\partial$ не являются $R$-линейно независимыми. Чтобы избежать этого, мы введем в рассмотрение алгебру $R[\xi]$, умножение в которой определяется практически так же, как и в $R[\partial]$, но соответствующие отношения в которой разделены. Элементами $R[\xi]$ являются формальные выражения

$$
\sum_{i=0}^{n} a_{i} \xi^{i}, \quad a_{i} \in R, \quad i \geqslant 0 .
$$

Их сложение, умножение, а также умножение на скаляры заданы следующим обра30M:

$$
\begin{aligned}
\sum_{i} a_{i} \xi^{i}+\sum_{i} b_{i} \xi^{i} & =\sum_{i}\left(a_{i}+b_{i}\right) \xi^{i}, \\
\left(\sum_{i=0}^{n} a_{i} \xi^{i}\right)\left(\sum_{j=0}^{m} b_{j} \xi^{i}\right) & =\sum_{\substack{0 \leqslant i \leqslant n, 0 \leqslant k \leqslant i \\
0 \leqslant j \leqslant m}} \sum_{\substack{i \\
0 \leqslant}}\left(\begin{array}{l}
i \\
k
\end{array}\right) a_{i} \partial^{k}\left(b_{j}\right) \xi^{i+j-k}, \\
\lambda . a=\lambda .\left(\sum_{j} a_{j} \xi^{j}\right) & :=\sum_{j} \lambda a_{j} \xi^{j} .
\end{aligned}
$$

Можно показать, что такая мультипликативная структура ассоциативна, и поэтому $R[\xi]$ становится $k$-алгеброй, а также свободным модулем над кольцом $R$ с базисом $\left\{\xi^{i} \mid i \geqslant 0\right\}$. Отображение

$$
a=\sum_{i=0}^{n} a_{i} \xi^{i} \mapsto \phi(a):=\sum_{i=0}^{n} a_{i} \partial^{i}
$$

есть сюръективный гомоморфизм $\phi k$-алгебр, действующий из $R[\xi]$ на $R[\partial]$. В вырожденном случае, т. е. когда $\partial=0$, кольцо $R[\xi]$ превращается в коммутативную алгебру

$$
R[\lambda]=\left\{\sum_{i=0}^{n} a_{i} \lambda^{i} \mid a_{i} \in R\right\}
$$

многочленов от $\lambda$ с коэффициентами из $R$. Можно рассматривать $R[\xi]$ как деформацию коммутативной алгебры $R[\lambda]$. В некоторых работах (см., например, монографию [8]) предлагается работать напрямую с $R[\xi]$ вместо $R[\partial]$. Элементы $R[\xi]$ называют формальными дифференциальными операторами относительно $\partial$.

В $R[\xi]$ имеет смысл ввести понятие степени.

ОПРЕДЕЛЕНиЕ 1. Cтепень $\operatorname{deg}(a)$ ненулевого элемента $a=\sum_{i=0}^{N} a_{i} \xi^{i}$ из $R[\xi]$ есть число $N$, если $a_{N} \neq 0$. Степень нулевого элемента мы полагаем равной $-\infty$. 
Элементы $\xi^{m}, m \geqslant 1$, в $R[\xi]$ необратимы, поэтому мы построим расширение алгебры $R[\xi]$, которое будет содержать все формальные обратные $\left\{\xi^{-m} \mid m \geqslant 1\right\}$. Рассматривая элементы $R[\xi]$ как “дифференциальные операторы”, мы можем осуществить этот процесс, добавив в алгебру $R[\xi]$ "интегральные операторы", но проделав это чисто алгебраическим способом, как будет показано ниже. В этой расширенной алгебре для всех $m$ и $n \in \mathbb{Z}$ в первую очередь должно быть выполнено

$$
\xi^{n} \xi^{m}=\xi^{n+m} .
$$

Чтобы выяснить, как должен действовать $\xi^{-1}$ на элемент вида $b \xi^{m}, b \in R$, применим последовательно правило $\xi^{-1} c=c \xi^{-1}-\xi^{-1} \partial(c) \xi^{-1}$, которое приводит к предположению, что $\xi^{-1}\left(b \xi^{m}\right)$ имеет вид бесконечного ряда отрицательных степеней $\xi$ :

$$
\xi^{-1}\left(b \xi^{m}\right)=\sum_{s=0}^{\infty}(-1)^{s} \partial^{s}(b) \xi^{m-1-s} .
$$

Формула (19) определяет также действие других отрицательных степеней $\xi^{n}, n<0$, и, обозначив

$$
\left(\begin{array}{l}
n \\
k
\end{array}\right):=\frac{n(n-1) \cdots(n-k+1)}{k !},
$$

$n \in \mathbb{Z}$, можно доказать, используя индукцию по $|n|$, что действие $\xi^{n}$ определяется равенством

$$
\xi^{n} b \xi^{m}=\sum_{s=0}^{\infty}\left(\begin{array}{l}
n \\
s
\end{array}\right) \partial^{s}(b) \xi^{m+n-s} .
$$

Эта формула позволяет ввести мультипликативную структуру в множестве $R\left[\xi, \xi^{-1}\right.$ ) всех формальных степеннь́х рядов

$$
p=\sum_{j=-\infty}^{N} p_{j} \xi^{j}, \quad p_{j} \in R .
$$

Произведение двух таких рядов $a=\sum_{j} a_{j} \xi^{j}$ и $b=\sum_{i} b_{i} \xi^{i}$ определим как

$$
a . b:=\sum_{j} \sum_{i} \sum_{s=0}^{\infty}\left(\begin{array}{l}
j \\
s
\end{array}\right) a_{j} \partial^{s}\left(b_{i}\right) \xi^{i+j-s},
$$

что является очевидным расширением (17). Далее, если определить на $R\left[\xi, \xi^{-1}\right)$ сложение и умножение на числа из $k$ формулами (16) и (18) соответственно, то $R\left[\xi, \xi^{-1}\right)$ становится $k$-алгеброй. Эта алгебра, представляющая собой смесь дифференциальных и интегральных операторов, имеет специальное название.

ОПрЕДЕлЕНИЕ 2. Элементы $R\left[\xi, \xi^{-1}\right)$ называются псевдодифференииалъными операторами относительно д или просто псевдодифберенциалъными операторами, если не требуется особо отметить операцию дифференцирования, которая лежит в их основе. Для обозначения $k$-алгебры $R\left[\xi, \xi^{-1}\right)$ будем использовать аббревиатуру Psd.

Далее, понятие степени можно распространить и на псевдодифференциал. 
ОПРЕДЕЛЕНИЕ 3. Степенъю $\operatorname{deg}(a)$ ненулевого элемента $a$ из $R\left[\xi, \xi^{-1}\right)$ называется целое $n$, для которого

$$
a=a_{n} \xi^{n}+\sum_{j<n} a_{j} \xi^{j}, \quad a_{n} \neq 0 .
$$

Добавление элементов, обратных к $\left\{\xi^{m} \mid m \geqslant 1\right\}$, увеличивает количество обратимых элементов в $R\left[\xi, \xi^{-1}\right)$, поскольку имеет место следующая лемма.

Лемма 1. Каждый псевдодифференииальный оператор $P=\sum_{j \leqslant m} p_{j} \xi^{j}$, где $p_{m} \in R^{*}$, имеет обратный оператор $P^{-1}$ вида $\sum_{i \leqslant-m} q_{i} \xi^{i}$, где $q_{-m}=p_{m}^{-1}$.

ДоказАтельство. Произведение элементов $\sum_{j \leqslant m} p_{j} \xi^{j}$ и $\sum_{i \leqslant-m} q_{i} \xi^{i}$ по определению равно

$$
\sum_{j \leqslant m} p_{j} \sum_{i \leqslant-m} \sum_{s=0}^{\infty}\left(\begin{array}{l}
j \\
s
\end{array}\right) p_{j} \partial^{s}\left(q_{i}\right) \xi^{j+i-s} .
$$

Это произведение есть оператор неположительной степени, и если он равен 1 , то старший коэффициент $q_{-m}$ должен быть обратным элементом к $p_{m}$, и для всех $k \geqslant 1$ должно выполняться равенство

$$
\sum_{\substack{i, j, s, i+j-s=-k}}\left(\begin{array}{l}
j \\
s
\end{array}\right) p_{j} \partial^{s}\left(q_{i}\right)=p_{m} q_{-m-k}+\sum_{\substack{i, j, s, i+j-s=-k, i>-m-k}}\left(\begin{array}{l}
j \\
s
\end{array}\right) p_{j} \partial^{s}\left(q_{i}\right)=0 .
$$

Так как $p_{m}$ обратим, отсюда можно найти $q_{-m-k}$, предположив, что все коэффициенты $q_{i}, i>-m-k$, известны. Это доказывает существование оператора, обратного к $P$. Лемма доказана.

Поскольку алгебра $R\left[\xi, \xi^{-1}\right)$ имеет широкий спектр обратимых элементов, у нее есть большой потенциал для процедуры одевания.

ОПРЕДЕЛЕНИЕ 4. Говорят, что элемент $P \in R\left[\xi, \xi^{-1}\right)$ получается одеванием элемента $Q \in R\left[\xi, \xi^{-1}\right)$, если существует такой обратимый элемент $K \in R\left[\xi, \xi^{-1}\right)$, что $P=K Q K^{-1}$. Оператор $K$ в этом случае называется одевающим оператором.

В дальнейшем нам предстоит столкнуться с двумя подгруппами обратимых элементов $R\left[\xi, \xi^{-1}\right)$, которые заслуживают особого внимания, а именно с группой

$$
D(0)=\left\{p_{0}+\sum_{j<0} p_{j} \xi^{j} \mid p_{0} \in R^{*}\right\}
$$

и ее нормальной подгруппой $D(0)_{1}$ элементов вида $1+\sum_{j<0} p_{j} \xi^{j}$.

Как и любая ассоциативная $k$-алгебра, $R\left[\xi, \xi^{-1}\right)$ является алгеброй Ли над полем $k$ относительно коммутатора. Внутри алгебры Ли $R\left[\xi, \xi^{-1}\right)$ можно использовать различные разложения. Любой $P=\sum_{j} p_{j} \xi^{j} \in R\left[\xi, \xi^{-1}\right)$ может быть разложен в сумму двух его составляющих - дифференциального оператора $P_{\geqslant 0}$ и строго интегрального оператора $P_{<0}$, где

$$
P_{\geqslant 0}=\sum_{j \geqslant 0} p_{j} \xi^{j}, \quad P_{<0}=\sum_{j<0} p_{j} \xi^{j} .
$$

6 Теоретическая и математическая физика, т. 174, № 1, 2013 г. 
Аналогично оператор $P$ можно представить как сумму его чисто дифференциальной части $P_{>0}$ и интегральной части $P_{\leqslant 0}$ :

$$
P_{>0}=\sum_{j>0} p_{j} \xi^{j}, \quad P_{\leqslant 0}=\sum_{j \leqslant 0} p_{j} \xi^{j} .
$$

Из правил умножения, заданных в $R\left[\xi, \xi^{-1}\right)$, следует, что эти два разложения операторов определяют два способа разложения алгебры Ли $R\left[\xi, \xi^{-1}\right)$ в прямую сумму двух подалгебр Ли. Первый способ:

$$
\text { Psd }=\left\{P \in \operatorname{Psd}, P=P_{<0}\right\} \oplus\left\{P \in \operatorname{Psd}, P=P_{\geqslant 0}\right\}:=\operatorname{Psd}_{<0} \oplus \operatorname{Psd}_{\geqslant 0} ;
$$

второй способ:

$$
\text { Psd }=\{P \in \operatorname{Psd}, P=P \leqslant 0\} \oplus\left\{P \in \operatorname{Psd}, P=P_{>0}\right\}:=\operatorname{Psd}_{\leqslant 0} \oplus \operatorname{Psd}_{>0} .
$$

Предположение $\mathfrak{g}_{2}=\operatorname{Psd}_{\geqslant 0}$, как и предположение $\mathfrak{g}_{2}=\operatorname{Psd}_{>0}$, не позволяет удачно подобрать группу $G_{2}$. Тем не менее если $\mathfrak{g}_{1}=\operatorname{Psd}_{<0}$, то появляется подходящий вариант для группы $G_{1}$. А именно, для каждого элемента $P=\sum_{j<0} p_{j} \xi^{j} \in R\left[\xi, \xi^{-1}\right)_{<0}$ и любого $m \geqslant 1$ элемент $P^{m}$ имеет степень, меньшую либо равную $-m$. Тогда формула

$$
e^{P}=\sum_{m=0}^{\infty} \frac{P^{m}}{m !}
$$

определяет корректно заданный элемент из $D(0)_{1}$. Обратно, аналогичные рассуждения показывают, что для каждого элемента $P=\sum_{j<0} p_{j} \xi^{j} \in R\left[\xi, \xi^{-1}\right)_{<0}$ формула

$$
\ln (1+P):=\sum_{m=1}^{\infty}(-1)^{m+1} \frac{P^{m}}{m}
$$

определяет корректно заданный элемент из $R\left[\xi, \xi^{-1}\right)_{<0}$. Очевидно, что он удовлетворяет равенству $1+P=e^{\ln (1+P)}$. Таким образом, $D(0)_{1}$ можно рассматривать как группу $G_{1}$, соответствующую алгебре Ли $\mathfrak{g}_{1}=\operatorname{Psd}_{<0}$.

Для случая $\mathfrak{g}_{1}=\mathrm{Psd}_{\leqslant 0}$ можно рассмотреть, как и выше, экспоненциальное отображение элементов $P=\sum_{j \leqslant 0} p_{j} \xi^{j} \in R\left[\xi, \xi^{-1}\right)_{\leqslant 0}$, что требует, однако, учитывать сходимость, как это видно по члену нулевого порядка. Необходимо, чтобы для каждого $p_{0} \in R$ выполнялось $e^{p_{0}} \in R$. Таким образом, если экспоненциальное отображение дает корректно заданный элемент из $\mathrm{Psd}_{\leqslant 0}$, то он определяет элемент из $D(0)$. Следовательно, $D(0)$ может рассматриваться как группа, соответствующая $\operatorname{Psd}_{\leqslant 0}$.

\section{4. УРАВНЕНИЯ ЛАКСА ДЛЯ ПСЕВДОДИФФЕРЕНЦИАЛЬНЫХ ОПЕРАТОРОВ}

В настоящем разделе мы опишем совместные системы уравнений Лакса в $R\left[\xi, \xi^{-1}\right)$, которые являются аналогами конечномерных систем, рассмотренных в теореме 1 , и основаны на разложениях, представленных в разделе 3. Здесь появятся другие $k$-линейные операции дифференцирования $R$, коммутирующие с $\partial$, такие, как оператор $\partial / \partial t$ в уравнении (14). Они также действуют естественным образом на элементы Psd. Мы опишем это действие и будем использовать его в дальнейшем без 
дополнительных напоминаний. Итак, пусть $\Delta$ - еще одно $k$-линейное дифференцирование $R$, которое коммутирует с $\partial$. Тогда действие $\Delta$ можно распространить на $R\left[\xi, \xi^{-1}\right)$, полагая для каждого $P=\sum_{j} p_{j} \xi^{j} \in R\left[\xi, \xi^{-1}\right)$

$$
\Delta(P)=\sum_{j} \Delta\left(p_{j}\right) \xi^{j} .
$$

Поскольку $\Delta$ и $\partial$ коммутируют, непосредственная проверка показывает, что имеет место следующая лемма.

Лемма 2. Расширение $\Delta$ на $R\left[\xi, \xi^{-1}\right)$ есть $k$-линейное дифференцирование этой алгебры.

Очевидный выбор коммутирующих элементов в $\operatorname{Psd}_{>0}$ и $\operatorname{Psd}_{\geqslant 0}$ есть $\left\{\xi^{m} \mid m \geqslant 1\right\}$ и $\left\{\xi^{m} \mid m \geqslant 0\right\}$ соответственно. Поскольку деформации, которые мы имеем в виду для этих направлений, состоят в сопряжении с соответствующими элементами группы $G_{1}$, мы можем не учитывать элемент $\xi^{0}$ и ограничиться в обоих случаях деформированием $\left\{\xi^{m} \mid m \geqslant 1\right\}$.

Рассмотрим сначала деформации с группой $D(0)_{1}$. Сопряжение с элементом $D(0)_{1}$ дает множество $\left\{L^{m}, m \geqslant 1\right\}$, где $L=K \xi K^{-1}, K \in D(0)_{1}$, имеет вид

$$
L=\xi+\sum_{j=1}^{\infty} \ell_{j+1} \xi^{-j}
$$

При достаточно слабом условии любой $L$ вида $(27)$ может быть получен одеванием оператора $\xi$ элементами из $D(0)_{1}$.

ЛЕмма 3. Если Ә сюрбективно, то любой $P=\xi+\sum_{i=1}^{\infty} p_{i+1} \xi^{-i}$ может быть получен одеванием оператора $\xi$ элементом из $D(0)_{1}$.

ДокАЗАТЕЛьство. Доказательство заключается в пошаговом решении уравнения $P K=K \xi$ с $K \in D(0)_{1}$. Если $K=1+\sum_{j>0} k_{j} \xi^{-j}$, то его правая часть есть $\xi+\sum_{j>0} k_{j} \xi^{1-j}$, а левая часть равна

$$
\begin{aligned}
P K=( & \left.+\sum_{i=1}^{\infty} p_{i+1} \xi^{-i}\right)\left(1+\sum_{j>0} k_{j} \xi^{-j}\right)=\xi+\sum_{i>0} k_{i} \xi^{1-i}+ \\
& +\sum_{i>0} \partial\left(k_{i}\right) \xi^{-i}+\sum_{i=1}^{\infty} p_{i+1} \xi^{-i}+\sum_{i \geqslant 1} \sum_{j \geqslant 1} \sum_{l \geqslant 0} p_{i+1}\left(\begin{array}{c}
-i \\
l
\end{array}\right) \partial^{l}\left(k_{j}\right) \xi^{-i-j-l} .
\end{aligned}
$$

Отсюда следует, что мы должны выбрать $K$ так, чтобы

$$
\sum_{j \geqslant 1} \partial\left(k_{j}\right) \xi^{-j}+\sum_{i=1}^{\infty} p_{i+1} \xi^{-i}+\sum_{i \geqslant 1} \sum_{j \geqslant 1} \sum_{l \geqslant 0} p_{i+1}\left(\begin{array}{c}
-i \\
l
\end{array}\right) \partial^{l}\left(k_{j}\right) \xi^{-i-j-l}=0 .
$$

Коэффициент при $\xi^{-1}$ в выражении, стоящем в левой части, равен $\partial\left(k_{1}\right)+p_{2}$, и благодаря условию, наложенному на $\partial$, можно найти такой $k_{1}$, что этот коэффициент будет равен нулю. Предположим, что найдены $\left\{k_{1}, \ldots, k_{m}\right\}, m \geqslant 1$, такие, что коэффициенты при всех $\xi^{-l}, l \leqslant m$, равны нулю. Тогда следующий коэффициент должен иметь вид

$$
\partial\left(k_{m+1}\right)+p_{m+2}+\left(\text { многочлен относительно } \partial^{l}\left(k_{i}\right) \text { и } p_{j+1}\right), \quad i \leqslant m, \quad j \leqslant m,
$$

и можно выбрать $k_{m+1}$ так, что и он будет равен нулю. Таким образом, коэффициенты $K$ могут быть найдены по индукции. Лемма доказана. 
Деформации с $D(0)$ носят несколько более общий характер. Сопряжение базисных направлений с элементом из $D(0)$ дает множество $\left\{M^{m}, m \geqslant 1\right\}$, где оператор $M=D \xi D^{-1}, D \in D(0)$, имеет вид

$$
M=\xi+\sum_{j=0}^{\infty} m_{j+1} \xi^{-j} .
$$

Для того чтобы любой $M$ вида (29) можно было получить, одевая $\xi$ элементами из $D(0)$, помимо сюръективности $\partial$, необходимы дополнительные условия (ср. с леммой 3$)$.

Лемма 4. Пусть $R$ и д таковы, что д сюргективно и для каждого $r \in R$

$$
e^{r}=\sum_{i=0}^{\infty} \frac{r^{i}}{i !}
$$

есть корректно заданный элемент $R^{*}$, удовлетворяющий равенству $\partial\left(e^{r}\right)=\partial(r) e^{r}$. Тогда кажсдый элемент

$$
P=\xi+\sum_{i=0}^{\infty} p_{i+1} \xi^{-i}, \quad p_{0} \neq 0,
$$

может быть получен одеванием оператора $\xi$ элементом из $D(0)$.

ДокАзАтЕльство. Наша цель - найти оператор $D$ нулевого порядка с обратимым старшим коэффициентом такой, что $P D=D \xi$. Рассмотрим $D=k_{0} K$, где $k_{0}$ обратим, а $K$ есть оператор вида $K=1+K_{-}=1+\sum_{i \geqslant 1} k_{i} \xi^{-i}$. Если мы аналогичным образом представим оператор $P=\xi+p_{1}+P_{-}$, где $P_{-}=\sum_{i=1}^{\infty} p_{i+1} \xi^{-i}$, то согласно лемме 3 для того, чтобы решить уравнение $P k_{0} K=k_{0} K \xi$, достаточно найти $k_{0} \in R^{*}$ такой, что $k_{0}^{-1} P k_{0}$ не имеет постоянного члена. Прямые вычисления показывают, что $\xi^{0}$ в $k_{0}^{-1} P k_{0}$ равен $k_{0}^{-1} \partial\left(k_{0}\right)+p_{1}$; таким образом, мы должны решить уравнение $\partial\left(k_{0}\right)+p_{1} k_{0}=0$. Если $l_{1}$ есть первообразная $p_{1}$ относительно $\partial$, т. е. $\partial\left(l_{1}\right)=p_{1}$, то в качестве решения этого уравнения можно взять $k_{0}=e^{-l_{1}}$ из $R$. Лемма доказана.

Пример 2. Конкретными примерами $k$-алгебры $R$ и $k$-линейного дифференцирования $\partial$ на $R$, которые удовлетворяют условиям леммы 3 , являются $k=\mathbb{R}$ или $\mathbb{C}$, $R=k\left[\left[t_{i}, i \geqslant 1\right]\right]$ и $\partial:=\partial / \partial t_{1}$.

Теперь мы может обратиться к аналогам уравнений Лакса в теореме 1 для обоих разложений. Рассмотрим сначала $\mathfrak{g}_{1}=\operatorname{Psd}_{<0}$. Тогда имеем $\pi_{1}(P)=P_{<0}$. Каждому базисному направлению $\xi^{i}, i \geqslant 1$, в $\mathfrak{g}_{2}$ должен соответствовать инфинитезимальный образующий потока, т. е. $k$-линейное дифференцирование $\partial_{i}$ на $R$, которое коммутирует с $\partial$. Нам потребуются деформации $\left\{L^{m}, m \geqslant 1\right\}$ с $L$ вида (27), которые удовлетворяют уравнениям

$$
\partial_{i}\left(L^{m}\right)=\left[L^{m}, \pi_{1}\left(L^{i}\right)\right]=\left[L_{\geqslant 0}^{i}, L^{m}\right]=\left[B_{i}, L^{m}\right], \quad i \geqslant 1, \quad m \geqslant 1,
$$

где $B_{i}$ есть краткое обозначение для $L_{\geqslant 0}^{i}$. Поскольку и $\partial_{i}$, и коммутирование с $B_{i}$ оба являются операциями дифференцирования Psd, достаточно найти $L$, который при всех $i, i \geqslant 1$, удовлетворяет соотношениям

$$
\partial_{i}(L)=\left[B_{i}, L\right]=\left[L, \pi_{1}\left(L^{i}\right)\right] .
$$


Отметим, что последнее равенство означает, что $\left[B_{i}, L\right]$ есть оператор порядка $\leqslant-1$ как $\partial_{i}(L)$. Как мы увидим в дальнейшем, из этих уравнений следует, что дифференциальные операторы $\left\{B_{i}\right\}$ в $R\left[\xi, \xi^{-1}\right)$ удовлетворяют равенству

$$
\partial_{i_{1}}\left(B_{i_{2}}\right)-\partial_{i_{2}}\left(B_{i_{1}}\right)-\left[B_{i_{1}}, B_{i_{2}}\right]=0 .
$$

Пусть операции дифференцирования $\partial, \partial_{2}$ и $\partial_{3}$ суть

$$
\partial=\frac{\partial}{\partial x}, \quad \partial_{2}=\frac{\partial}{\partial t_{2}}, \quad \partial_{3}=\frac{\partial}{\partial t_{3}}
$$

соответственно. Тогда это уравнение при $i_{1}=3$ и $i_{2}=2$ сводится к уравнению

$$
3\left(\ell_{2}\right)_{t_{2} t_{2}}=\left(2\left(\ell_{2}\right)_{t_{3}}-\frac{1}{2}\left(\ell_{2}\right)_{x x x}-6 \ell_{2}\left(\ell_{2}\right)_{x}\right)_{x}
$$

для $\ell_{2}$, которое с точностью до коэффициента масштабирования представляет собой уравнение КП, и это объясняет следующую терминологию.

ОПредЕлЕниЕ 5 . Пусть задана $k$-алгебра $R$, наделенная преимущественной $k$ линейной операцией дифференцирования $\partial$, и множество $\left\{\partial_{i} \mid i \geqslant 1\right\} k$-линейных операций дифференцирования, коммутирующих с ә. Уравнения (31) для оператоpa $L$ из $R\left[\xi, \xi^{-1}\right)$ вида (27) называются уравнениями Лакса иерархии KП. $L$ будем называть решением иерархии, а набор $\left(R, \partial,\left\{\partial_{i} \mid i \geqslant 1\right\}\right)$ - реализацией этой нелинейной системы.

ЗАмЕчАниЕ 1. Отметим, что любая реализация иерархии КП допускает тривиальное решение $L=\xi$.

Далее, возьмем $\mathfrak{g}_{1}=\operatorname{Psd}_{\leqslant 0}$. Тогда $\pi_{1}(P)=P_{\leqslant 0}$. Снова для каждого $r, r \geqslant 1$, нам нужен инфинитезимальный образующий потока, который соответствует каждому базисному направлению $\xi^{r}$. Чтобы отличать данный случай от вышеизложенного, обозначим такое $k$-линейное дифференцирование, которое коммутирует с $\partial$, через $\underline{\partial}_{r}$. Здесь нам нужны деформации $\left\{M^{m}, m \geqslant 1\right\}$ с $M$ вида (29), удовлетворяющие уравнениям

$$
\underline{\partial}_{r}\left(M^{m}\right)=\left[M^{m}, \pi_{1}\left(M^{r}\right)\right]=\left[M_{>0}^{r}, L^{m}\right]=\left[C_{r}, M^{m}\right], \quad r \geqslant 1, \quad m \geqslant 1,
$$

где $C_{r}$ есть краткое обозначение для $M_{>0}^{r}$. Поскольку и $\underline{\partial}_{r}$, и коммутирование с $C_{r}$ являются операциями дифференцирования $\mathrm{Psd}$, достаточно найти $M$, удовлетворяющий для всех $r, r \geqslant 1$, соотношениям

$$
\underline{\partial}_{r}(M)=\left[C_{r}, M\right]=\left[M, \pi_{1}\left(M^{r}\right)\right] .
$$

Отметим, что последнее равенство указывает на то, что $\left[C_{r}, M\right]$ есть оператор порядка $\leqslant 0$ как $\underline{\partial}_{r}(M)$. Поскольку иерархия КП базируется на разложении, в котором $\pi_{2}(P)$ является той частью псевдодифференциального оператора $P$, которая представляет собой полный дифференциальный оператор, а настоящее разложение есть его строгая версия, мы используем следующую терминологию.

ОПРЕДЕЛЕниЕ 6 . Пусть задана $k$-алгебра $R$, наделенная преимущественной $k$ линейной операцией дифференцирования $\partial$, и множество $\left\{\underline{\partial}_{r} \mid r \geqslant 1\right\} k$-линейных операций дифференцирования, коммутирующих с д. Уравнения (34) для оператора $M$ из $R\left[\xi, \xi^{-1}\right)$ вида (29) называются уравнениями Лакса строгой иерархии КП. $M$ будем называть решением иерархии, а набор $\left(R, \partial,\left\{\partial_{r} \mid r \geqslant 1\right\}\right)$ - реализацией этой нелинейной системы. 
ЗАмЕчАНИЕ 2. Отметим, что любая реализация строгой иерархии КП также допускает тривиальное решение $M=\xi$.

Как было замечено ранее, каждый $\left[C_{r}, M\right]$ есть оператор порядка $\leqslant 0$. Аналогично каждый $\left[B_{i}, L\right]$ есть оператор порядка $\leqslant-1$. Предположим, что $Q \in R[\xi]$ имеет степень $k \geqslant 1$, не имеет постоянного члена, а степень $[Q, M]$ не превышает нуля. Пусть также $P$ есть элемент $R[\xi]$, коммутатор которого с $L$ имеет порядок $\leqslant-1$. Тогда имеет смысл рассмотреть $k$-линейное дифференцирование $\underline{\partial}_{Q}$ на $R$ такое, что

$$
\underline{\partial}_{Q}(M)=[Q, M],
$$

и $k$-линейное дифференцирование $\partial_{P}$ на $R$ такое, что

$$
\partial_{P}(L)=[P, L] .
$$

Следующее утверждение показывает, что это не добавит ничего нового к уравнениям, рассматриваемым как в случае иерархии КП, так и в случае строгой иерархии КП.

УтВЕРЖДЕНИЕ 1. Справедливы следующие утверждения:

1) пусть $Q \in R[\xi]$ есть элемент степени $k \geqslant 1$ без постоянного члена такой, что степень коммутатора $[Q, M]$ не превышает нуля; тогда существует единственный набор элементов $c_{r} \in R, \partial\left(c_{r}\right)=0,1 \leqslant r \leqslant k, \operatorname{ma\kappa ux,~что~} Q=\sum_{r=1}^{k} c_{r} C_{r}$;

2) пусть $P \in R[\xi]$ есть элемент степени $k$ такой, что коммутатор $[P, L]$ имеет отрицательную степенъ; тогда существует единственный набор элементов $p_{i} \in R, 0 \leqslant i \leqslant k, \partial\left(p_{i}\right)=0$, maкux, ито $P=\sum_{i=0}^{k} p_{i} B_{i}$.

ДоКАЗАТЕЛьСтво. Для доказательства используем индукцию по степени $Q$. Если степень $Q$ равна 1 , то $Q=c_{1} \xi$, где степень $\left[c_{1} \xi, M\right]$ не превышает нуля. Это означает, в частности, что единственный член степени 1 , полученный посредством коммутатора $[Q, M]$, а именно $\left[c_{1} \xi, \xi\right]=-\partial\left(c_{1}\right) \xi$, должен быть равен нулю. Тогда $Q=c_{1} C_{1}$ с $\partial\left(c_{1}\right)=0$. Пусть теперь $Q=c_{k+1} \xi^{k+1}+R_{k}$, где $R_{k}$ имеет степень $\leqslant k$. Тогда получаем $\left[c_{k+1} \xi^{k+1}, \xi\right]=-\partial\left(c_{k+1}\right) \xi^{k+1}$, и это единственный член в $[Q, L]$, имеющий степень $k+1$. Следовательно, $\partial\left(c_{k+1}\right)$ должен быть равен нулю. Оператор $Q-c_{k+1} C_{k+1}$ имеет степень $\leqslant k$, не содержит постоянного члена и обладает тем же свойством, что и $Q$; значит, он имеет вид

$$
Q-c_{k+1} C_{k+1}=\sum_{i=1}^{k} c_{i} C_{i}, \quad \partial\left(c_{i}\right)=0 .
$$

Таким образом, первое утверждение доказано; второе доказывается аналогично.

Следующий раздел посвящен минимальному способу реализации уравнений Лакса для обеих иерархий.

\section{5. МИНИМАЛЬНАЯ РЕАЛИЗАЦИЯ УРАВНЕНИЙ ЛАКСА}

Здесь мы хотим реализовать уравнения (31) и (34) с минимальным количеством соотношений между коэффициентами потенциальных решений $M$ и $L$ соответственно и их производных относительно д. Мы формализуем это следующим образом: в качестве алгебры $R$ в случае строгой иерархии КП мы рассмотрим алгебру

$$
\underline{\widetilde{R}}:=k\left[\widetilde{m}_{j+1}^{(s)} \mid j \geqslant 0, s \geqslant 0\right]
$$


всех многочленов от неизвестных $\left\{\widetilde{m}_{j+1}^{(s)} \mid j \geqslant 0, s \geqslant 0\right\}$ с коэффициентами из $k$, а в КП-случае - алгебру

$$
\widetilde{R}:=k\left[\tilde{\ell}_{j+1}^{(s)} \mid j \geqslant 1, s \geqslant 0\right]
$$

всех многочленов от неизвестных $\left\{\tilde{\ell}_{j+1}^{(s)} \mid j \geqslant 1, s \geqslant 0\right\}$ с коэффициентами из $k$. Напомним, что $k$-линейное дифференцирование $\Delta$ кольца многочленов $k\left[x_{s}\right]$ любого количества переменных определяется однозначно заданием образов $\Delta\left(x_{s}\right)$ всех $\left\{x_{s}\right\}$ в силу свойства дифференцирования

$$
\Delta(f g)=\Delta(f) g+f \Delta(g)
$$

для любых $f$ и $g \in k\left[x_{s}\right]$. Более того, $\Delta\left(x_{s}\right)$ можно выбирать произвольным образом. С учетом этого преимущественное дифференцирование $\underline{\tilde{\partial}}$ в первом случае определено как

$$
\underline{\tilde{\partial}}\left(\widetilde{m}_{j+1}^{(s)}\right)=\widetilde{m}_{j+1}^{(s+1)}, \quad j \geqslant 0, \quad s \geqslant 0 .
$$

Аналогично базисное дифференцирование $\tilde{\partial}$ на $\widetilde{R}$ можно определить равенством

$$
\tilde{\partial}\left(\tilde{\ell}_{i+1}^{(s)}\right)=\tilde{\ell}_{i+1}^{(s+1)}, \quad i \geqslant 1, \quad s \geqslant 0 .
$$

Таким образом, начиная с псевдодифференциальных операторов $\widetilde{M}$ и $\widetilde{L}$, определяемых как

$$
\widetilde{M}:=\xi+\sum_{j=0}^{\infty} \widetilde{m}_{j+1}^{(0)} \xi^{-j}, \quad \widetilde{L}:=\xi+\sum_{j=1}^{\infty} \tilde{\ell}_{j+1}^{(0)} \xi^{-j}
$$

соответственно, можно заметить, что верхний индекс измеряет, сколько раз должны подействовать $\underline{\tilde{\partial}}$ и $\tilde{\partial}$ на коэффициенты $\widetilde{M}$ и $\widetilde{L}$ соответственно. Очевидно, что здесь нет связей между коэффициентами $\widetilde{M}$ и $\widetilde{L}$ и их производными относительно $\underline{\tilde{\partial}}$ и $\tilde{\partial}$ соответственно.

Далее, мы хотим определить $k$-дифференцирования $\left\{\underline{\tilde{\partial}}_{r} \mid r \geqslant 1\right\}$ на $\underline{\widetilde{R}}$, которые коммутируют с $\underline{\tilde{\partial}}$. Это последнее свойство приводит к

$$
\underline{\tilde{\partial}}_{r}\left(\widetilde{m}_{j+1}^{(s)}\right)=\underline{\tilde{\partial}}^{s} \underline{\tilde{\partial}}_{r}\left(\widetilde{m}_{j+1}^{(0)}\right)
$$

и, следовательно, достаточно определить, как действует $\underline{\partial}_{r}$ на коэффициенты $\widetilde{m}_{j+1}^{(0)}$ у $\widetilde{M}$. Это можно сделать оптимальным способом в терминах отношения, которому должен удовлетворять $\widetilde{M}$. В соответствии с предыдущим разделом обозначим оператор $\widetilde{M}_{>0}^{r}$ для каждого $r \geqslant 1$ через $\widetilde{C}_{r}$. Далее, дифференцирование $\underline{\tilde{\partial}}_{r}$ на $\underline{\widetilde{R}}$ определим следующим равенством внутри $\underline{\widetilde{R}}\left[\xi, \xi^{-1}\right)$ :

$$
\underline{\tilde{\partial}}_{r}(\widetilde{M})=\sum_{j=0}^{\infty} \tilde{\tilde{\partial}}_{r}\left(\widetilde{m}_{j+1}^{(0)}\right) \xi^{-j}:=\left[\widetilde{C}_{r}, \widetilde{M}\right]=\left[\widetilde{M}, \widetilde{M}_{\leqslant 0}^{r}\right] .
$$

Аналогично можно определить множество $k$-дифференцирований $\left\{\tilde{\partial}_{i} \mid i \geqslant 1\right\}$ на $\widetilde{R}$, коммутирующих с $\tilde{\partial}$, задавая их действие на коэффициенты $\widetilde{L}$ в терминах набора отношений для $\widetilde{L}$. Как и прежде, для каждого $i \geqslant 1$ будем использовать обозначение $\widetilde{B}_{i}$ для псевдодифференциального оператора $\widetilde{L}_{\geqslant 0}^{i}$. Тогда уравнения, которым должен удовлетворять оператор $\widetilde{L}$, будут иметь вид

$$
\tilde{\partial}_{i}(\widetilde{L})=\sum_{j=1}^{\infty} \tilde{\partial}_{i}\left(\tilde{\ell}_{j+1}^{(0)}\right) \xi^{-j}:=\left[\widetilde{B}_{i}, \widetilde{L}\right]=\left[\widetilde{L}, \widetilde{L}_{<0}^{i}\right] .
$$


В обоих случаях уравнения Лакса выполнены по определению. Тем не менее из них можно извлечь некоторые следствия. Первое касается ряда нелинейных уравнений для множеств дифференциальных операторов $\left\{\widetilde{C}_{r}\right\}$ и $\left\{\widetilde{B}_{i}\right\}$ соответственно.

УтвеРжДЕниЕ 2. 1. Дифференциальные операторы $\left\{\widetilde{C}_{r}\right\}$ в $\underline{\widetilde{R}}\left[\xi, \xi^{-1}\right)$ удовлетворяют уравнениям

$$
\underline{\tilde{\partial}}_{r_{1}}\left(\widetilde{C}_{r_{2}}\right)-\underline{\tilde{\partial}}_{r_{2}}\left(\widetilde{C}_{r_{1}}\right)-\left[\widetilde{C}_{r_{1}}, \widetilde{C}_{r_{2}}\right]=0,
$$

которые называются отношениями нулевой кривизны решений $\widetilde{M}$ строгой иерархии КП.

2. Дифференциальные операторы $\left\{\widetilde{B}_{i}\right\}$ в $\widetilde{R}\left[\xi, \xi^{-1}\right)$ удовлетворяют уравнениям

$$
\tilde{\partial}_{i_{1}}\left(\widetilde{B}_{i_{2}}\right)-\tilde{\partial}_{i_{2}}\left(\widetilde{B}_{i_{1}}\right)-\left[\widetilde{B}_{i_{1}}, \widetilde{B}_{i_{2}}\right]=0,
$$

которые называются отношениями нулевой кривизны решений $\widetilde{L}$ иерархии КП.

ДокАЗАТЕЛЬСТво. Первое утверждение будет справедливым, если мы сможем доказать, что левая часть уравнения (37) принадлежит одновременно и $\underline{\widetilde{R}}\left[\xi, \xi^{-1}\right)_{>0}$, и $\underline{\widetilde{R}}\left[\xi, \xi^{-1}\right)_{\leqslant 0}$. Первое очевидно, так как все $\widetilde{C}_{r}$ принадлежат $\underline{\widetilde{R}}\left[\xi, \xi^{-1}\right)_{>0}$, а $\underline{\tilde{\partial}}_{r}$ действуют покоэффициентно. Как было отмечено ранее, из уравнений Лакса для $\widetilde{M}$ следуют уравнения Лакса для его степеней

$$
\underline{\tilde{Q}}_{r_{1}}\left(\widetilde{M}^{r_{2}}\right)=\left[\widetilde{C}_{r_{1}}, \widetilde{M}^{r_{2}}\right]=-\left[\widetilde{M}_{<0}^{r_{1}}, \widetilde{M}^{r_{2}}\right] .
$$

Далее, заменим в левой части уравнения (37)

$$
\widetilde{C}_{r_{k}}=\widetilde{M}^{r_{k}}-\widetilde{M}_{\leqslant 0}^{r_{k}}
$$

для $k=1,2$ и воспользуемся соотношениями (39). Получим

$$
\begin{gathered}
\underline{\tilde{\partial}}_{r_{1}}\left(\widetilde{C}_{r_{2}}\right)-\underline{\tilde{\partial}}_{r_{2}}\left(\widetilde{C}_{r_{1}}\right)-\left[\widetilde{C}_{r_{1}}, \widetilde{C}_{r_{2}}\right]=\left[\widetilde{C}_{r_{1}}, \widetilde{M}^{r_{2}}\right]-\tilde{\underline{\partial}}_{r_{1}}\left(\widetilde{M}_{\leqslant 0}^{r_{2}}\right)-\left[\widetilde{C}_{r_{2}}, \widetilde{M}^{r_{1}}\right]+ \\
+\widetilde{\tilde{\partial}}_{r_{2}}\left(\widetilde{M}_{\leqslant 0}^{r_{1}}\right)+\left[\widetilde{M}_{\leqslant 0}^{r_{1}}, \widetilde{M}^{r_{2}}\right]-\left[\widetilde{M}^{r_{1}}, \widetilde{M}_{\leqslant 0}^{r_{2}}\right]-\left[\widetilde{M}_{<0}^{r_{1}}, \widetilde{M}_{\leqslant 0}^{r_{2}}\right]= \\
=\underline{\tilde{\partial}}_{r_{2}}\left(\widetilde{M}_{\leqslant 0}^{r_{1}}\right)-\underline{\tilde{\partial}}_{r_{1}}\left(\widetilde{M}_{\leqslant 0}^{r_{2}}\right)-\left[\widetilde{M}_{\leqslant 0}^{r_{1}}, \widetilde{M}_{\leqslant 0}^{r_{2}}\right] ;
\end{gathered}
$$

здесь последнее выражение очевидно принадлежит $\underline{\widetilde{R}}\left[\xi, \xi^{-1}\right) \leqslant 0$. Рассуждая аналогичным образом, можно доказать второе утверждение.

Следствием утверждения 2 является свойство, объединяющее потоки, которые принадлежат одной иерархии

Следствие 1. 1. Операции дифференцирования $\left\{\underline{\tilde{\partial}}_{r}\right\}$ на $\underline{\widetilde{R}}$ коммутируют не только с $\underline{\tilde{\partial}}$, но и между собой.

2. Операции дифференцирования $\left\{\tilde{\partial}_{i}\right\}$ на $\widetilde{R}$ коммутируют не только с $\tilde{\partial}$, но и между собой.

ДОКАЗАТЕЛЬСТво. Мы должны показать, что для любых индексов выполнены равенства

$$
\underline{\tilde{\partial}}_{r_{1}} \circ \underline{\tilde{\partial}}_{r_{2}}=\underline{\tilde{\partial}}_{r_{2}} \circ \underline{\tilde{\partial}}_{r_{1}}, \quad \tilde{\partial}_{i_{1}} \circ \tilde{\partial}_{i_{2}}=\tilde{\partial}_{i_{2}} \circ \tilde{\partial}_{i_{1}} .
$$

Поскольку $\left\{\underline{\tilde{\partial}}_{r}\right\}$ и $\left\{\tilde{\partial}_{i}\right\}$ коммутируют с $\underline{\tilde{\partial}}$ и $\tilde{\partial}$ соответственно, то же самое должно выполняться и для их произведений. Следовательно, если их разности равны нулю 
на коэффициентах $\widetilde{M}$ и $\widetilde{L}$ соответственно, то они являются тождественными нулями на всем $\underline{\widetilde{R}}$ и $\widetilde{R}$. Таким образом, достаточно показать, что

$$
\underline{\tilde{\partial}}_{r_{1}} \circ \underline{\tilde{\partial}}_{r_{2}}(\widetilde{M})=\underline{\tilde{\partial}}_{r_{2}} \circ \underline{\tilde{\partial}}_{r_{1}}(\widetilde{M}), \quad \tilde{\partial}_{i_{1}} \circ \tilde{\partial}_{i_{2}}(\widetilde{L})=\tilde{\partial}_{i_{2}} \circ \tilde{\partial}_{i_{1}}(\widetilde{L})
$$

соответственно. Что касается первого соотношения, мы воспользуемся уравнениями Лакса для $\widetilde{M}$ и тем фактом, что каждый $\underline{\tilde{\partial}}_{r_{k}}$ есть дифференцирование $\underline{\widetilde{R}}\left[\xi, \xi^{-1}\right)$, для получения уравнения

$\underline{\tilde{\partial}}_{r_{1}} \circ \underline{\tilde{\partial}}_{r_{2}}-\underline{\tilde{\partial}}_{r_{2}} \circ \underline{\tilde{\partial}}_{r_{1}}(\widetilde{M})=\left[\underline{\tilde{\partial}}_{r_{1}}\left(\widetilde{C}_{r_{2}}\right), \widetilde{M}\right]+\left[\widetilde{C}_{r_{2}},\left[\widetilde{C}_{r_{1}}, \widetilde{M}\right]\right]-\left[\underline{\partial}_{r_{2}}\left(\widetilde{C}_{r_{1}}\right), \widetilde{M}\right]-\left[\widetilde{C}_{r_{1}},\left[\widetilde{C}_{r_{2}}, \widetilde{M}\right]\right]$.

Поскольку коммутатор удовлетворяет равенству

$$
\left[\widetilde{C}_{r_{2}},\left[\widetilde{C}_{r_{1}}, \widetilde{M}\right]\right]-\left[\widetilde{C}_{r_{1}},\left[\widetilde{C}_{r_{2}}, \widetilde{M}\right]\right]=\left[\left[\widetilde{C}_{r_{2}}, \widetilde{C}_{r_{1}}\right], \widetilde{M}\right]
$$

то согласно первой части утверждения 2 имеем

$$
\left[\underline{\tilde{\partial}}_{r_{1}}\left(\widetilde{C}_{r_{2}}\right)-\underline{\tilde{\partial}}_{r_{2}}\left(\widetilde{C}_{r_{1}}\right)-\left[\widetilde{C}_{r_{1}}, \widetilde{C}_{r_{2}}\right], \widetilde{M}\right]=[0, \widetilde{M}]=0 .
$$

Это завершает доказательство для $\widetilde{M}$; доказательство для $\widetilde{L}$ аналогично.

\section{6. ОТНОШЕНИЯ НУЛЕВОЙ КРИВИЗНЫ}

Цель настоящего раздела - дать алгебраическое описание других реализаций решений обоих иерархий. Отправной точкой в обоих случаях являются операторы $\widetilde{M}$ и $\widetilde{L}$, представленные в разделе 5 . Пусть $\underline{R}$ и $R$ - другие $k$-алгебры, наделенные преимущественными $k$-линейными операциями дифференцирования $\underline{\partial}$ и $\partial$ соответственно. Далее рассмотрим предположительные решения

$$
M=\xi+\sum_{j=0}^{\infty} m_{j+1} \xi^{-j}, \quad L=\xi+\sum_{j=1}^{\infty} \ell_{j+1} \xi^{-j}
$$

в $\underline{R}\left[\xi, \xi^{-1}\right)$ и $R\left[\xi, \xi^{-1}\right)$ и соответствующие отсечения

$$
C_{r}:=M_{>0}^{r}, \quad r \geqslant 1, \quad B_{i}:=L_{\geqslant 0}^{i}, \quad i \geqslant 1 .
$$

Тогда $M$ однозначно определяет морфизм $k$-алгебр $i_{M}: \underline{\widetilde{R}} \rightarrow \underline{R}$ заданием

$$
i_{M}\left(\widetilde{m}_{j+1}^{(s)}\right)=\underline{\partial}^{s}\left(m_{j+1}\right),
$$

и этот морфизм $k$-алгебр по определению удовлетворяет равенству

$$
i_{M} \circ \underline{\tilde{\partial}}=\underline{\partial} \circ i_{M} \cdot
$$

Аналогично оператор $L$ определяет морфизм $k$-алгебр $i_{L}: \widetilde{R} \rightarrow R$ заданием

$$
i_{L}\left(\tilde{\ell}_{j+1}^{(s)}\right)=\partial^{s}\left(\ell_{j+1}\right),
$$

и этот морфизм $k$-алгебр по определению удовлетворяет равенству

$$
i_{L} \circ \tilde{\partial}=\partial \circ i_{L}
$$

Отображения $i_{M}$ и $i_{L}$ можно продолжить до морфизмов $k$-алгебр, действующих из множеств псевдодифференциальных операторов $\underline{\widetilde{R}}\left[\xi, \xi^{-1}\right)$ и $\widetilde{R}\left[\xi, \xi^{-1}\right)$, рассмотренных в разделе 5 , в $\underline{R}\left[\xi, \xi^{-1}\right)$ и $R\left[\xi, \xi^{-1}\right)$ соответственно таким образом, что

$$
i_{M}(\widetilde{M})=M, \quad i_{L}(\widetilde{L})=L .
$$


Для того чтобы можно было говорить о решениях иерархий, внутри $\underline{R}$ и $R$ должны существовать аналоги семейств $\left\{\underline{\tilde{\partial}}_{r}\right\}$ и $\left\{\tilde{\partial}_{i}\right\}$ соответственно. Это означает, что нужно определить множества $k$-линейных операций дифференцирования $\left\{\underline{\partial}_{r}\right\}$ и $\left\{\partial_{i}\right\}$ на $\underline{R}$ и $R$ соответственно, которые коммутируют с $\underline{\partial}$ и $\partial$. Предположив, что такие отображения существуют, теперь имеет смысл проверить, удовлетворяют ли $M$ и $L$ уравнениям Лакса относительно этих наборов операций дифференцирования. Итак, если $M$ есть решение уравнений Лакса для строгой иерархии КП, то для всех $r \geqslant 1$ мы имеем

$$
\underline{\partial}_{r}(M)=\underline{\partial}_{r} \circ i_{M}(\widetilde{M})=\left[C_{r}, M\right]=\left[i_{M}\left(\widetilde{C}_{r}\right), i_{M}(\widetilde{M})\right]=i_{M}\left(\left[\widetilde{C}_{r}, \widetilde{M}\right]\right)=i_{M} \circ \underline{\partial}_{r}(\widetilde{M}) .
$$

Таким образом, $k$-линейные отображения $\underline{\partial}_{r} \circ i_{M}$ и $i_{M} \circ \underline{\tilde{\partial}}_{r}$ совпадают на коэффициентах $\widetilde{M}$, а в силу соотношения (41) и того факта, что $\left\{\underline{\partial}_{r}\right\}$ коммутируют с $\partial$, мы получаем на $\underline{\tilde{R}}\left[\xi, \xi^{-1}\right)$ совместимости

$$
\underline{\partial}_{r} \circ i_{M}=i_{M} \circ \underline{\tilde{\partial}}_{r}, \quad r \geqslant 1 .
$$

С другой стороны, если совместимости (44) имеют место, то эти тождества можно применить к $\widetilde{M}$, и поскольку $i_{M}$ есть морфизм $k$-алгебр, получить уравнения Лакса для $M$. Таким образом, соотношения (44) равносильны тому факту, что $M$ является решением строгой иерархии КП относительно $\left\{\underline{\partial}_{r}\right\}$.

Существует схожая формулировка и того утверждения, что $L$ является решением иерархии КП. Действительно, если $L$ есть решение уравнений Лакса иерархии КП, то для любого $i \geqslant 1$ имеет место соотношение

$$
\partial_{i}(L)=\partial_{i} \circ i_{L}(\widetilde{L})=\left[B_{i}, L\right]=\left[i_{L}\left(\widetilde{B}_{i}\right), i_{L}(\widetilde{L})\right]=i_{L}\left(\left[\widetilde{B}_{i}, \widetilde{L}\right]\right)=i_{L} \circ \tilde{\partial}_{i}(\widetilde{L}) .
$$

Тогда $k$-линейные отображения $\partial_{i} \circ i_{L}$ и $i_{L} \circ \tilde{\partial}_{i}$ равны на коэффициентах $\widetilde{L}$, и, используя рассуждения, аналогичные случаю $M$, получаем соотношения

$$
\partial_{i} \circ i_{L}=i_{L} \circ \tilde{\partial}_{i}, \quad i \geqslant 1,
$$

на $\widetilde{R}\left[\xi, \xi^{-1}\right)$. Обратно, если выполнены соотношения (45), то, применяя эти тождества к $\widetilde{L}$ и учитывая, что $i_{L}$ есть морфизм $k$-алгебр, получаем уравнения Лакса для $L$. Таким образом, соотношения (45) эквиваленты тому, что $L$ суть решения иерархии КП относительно $\left\{\partial_{i}\right\}$.

Далее мы рассмотрим аналоги отношений нулевой кривизны (37) и (38) для отсечений $\left\{C_{r}\right\}$ и $\left\{B_{i}\right\} M$ и $L$ соответственно. Если мы совместим тождества (44) и (45) с результатом утверждения 2, то увидим, прежде всего, что строгие дифференциальные операторы $\left\{C_{r}\right\}$ в $\underline{R}\left[\xi, \xi^{-1}\right)$, отвечающие решению $M$ строгой иерархии КП, удовлетворяют уравнениям

$$
\underline{\partial}_{r_{1}}\left(C_{r_{2}}\right)-\underline{\partial}_{r_{2}}\left(C_{r_{1}}\right)-\left[C_{r_{1}}, C_{r_{2}}\right]=0
$$

Используя ту же терминологию, что и для минимальной реализации, будем называть уравнения (46) отношениями нулевой кривизны для строгих отсечений $\left\{C_{r}\right\}$ решения $M$ строгой иерархии КП. Во-вторых, для решения $L$ иерархии КП получаем, что дифференциальные операторы $\left\{B_{i}\right\}$ в $R\left[\xi, \xi^{-1}\right)$ удовлетворяют уравнениям

$$
\partial_{i_{1}}\left(B_{i_{2}}\right)-\partial_{i_{2}}\left(B_{i_{1}}\right)-\left[B_{i_{1}}, B_{i_{2}}\right]=0 .
$$


Здесь мы используем ту же терминологию, что и в минимальном случае, и назовем уравнения (47) отношениями нулевой кривизны дифференциальных операторов $\left\{B_{i}\right\}$, отвечающих решению $L$ иерархии КП.

Отношения нулевой кривизны в обоих случаях являются также достаточными для того, чтобы получить уравнения Лакса для $M$ и $L$, а именно имеет место

ТЕОРема 2. Пусть $\underline{R}$ u $R$ суть $k$-алгебры с преимущественными $k$-линейнъми операциями дифференцирования $\underline{\partial}$ и $\partial$ соответственно, и пусть $M$ и L суть элементы $\underline{R}\left[\xi, \xi^{-1}\right)$ и $R\left[\xi, \xi^{-1}\right)$ вида (29) и (27) соответственно.

1. Предположим, что в $\underline{R}$ существует множество $k$-линейных дифферениирований $\left\{\underline{\partial}_{r}, r \geqslant 1\right\}$, которые коммутируют с $\underline{\partial}$. Тогда $M$ удовлетворяет уравнениям Лакса строгой иерархии КП тогда и только тогда, когда для $\left\{C_{r}, r \geqslant 1\right\}$ выполненъ отношения нулевой кривизны (46).

2. Предположим, что в $R$ существует множество $k$-линейных дифферениирований $\left\{\partial_{i}, i \geqslant 1\right\}$, которые коммутируют с д. Тогда $L$ удовлетворяет уравнениям Лакса иерархии КП относительно $\left\{\partial_{i}\right\}$ тогда и только тогда, когда для $\left\{B_{i}, i \geqslant 1\right\}$ выполнены отношения нулевой кривизны (47).

ДокАЗАТЕЛьство. В обоих случаях нужно показать лишь достаточность. Мы приведем доказательство только первого утверждения, так как для второго оно абсолютно аналогично. Рассмотрим оператор $M$, для которого при всех $r_{1}$ и $r_{2}$ выполнено

$$
\underline{\partial}_{r_{1}}\left(C_{r_{2}}\right)-\underline{\partial}_{r_{2}}\left(C_{r_{1}}\right)-\left[C_{r_{1}}, C_{r_{2}}\right]=0 .
$$

Если при всех $r_{2} \geqslant 1$ мы запишем для простоты $M^{r_{2}}=C_{r_{2}}+D_{r_{2}}$, то это соотношение приведет к

$$
\begin{aligned}
\underline{\partial}_{r_{1}}\left(M^{r_{2}}\right)-\left[C_{r_{1}}, M^{r_{2}}\right] & =\underline{\partial}_{r_{1}}\left(C_{r_{2}}+D_{r_{2}}\right)-\left[C_{r_{1}}, C_{r_{2}}\right]-\left[C_{r_{1}}, D_{r_{2}}\right]= \\
& =\underline{\partial}_{r_{2}}\left(C_{r_{1}}\right)+\underline{\partial}_{r_{1}}\left(D_{r_{2}}\right)-\left[C_{r_{1}}, D_{r_{2}}\right] .
\end{aligned}
$$

Поскольку $D_{r_{2}}$ содержит отрицательные степени $\xi$, последнее выражение содержит степени $\xi$, не превышающие $r_{1}-2$ для всех $r_{2} \geqslant 1$. Предположим, что для некоторого $r_{1}$ уравнения Лакса относительно соответствующего дифференцирования не имеют места для $L$, т. е.

$$
\underline{\partial}_{r_{1}}(M)-\left[C_{r_{1}}, M\right]=\beta \xi_{m}+(\text { более низкий порядок } \xi), \quad \beta \neq 0 .
$$

Поскольку и $\underline{\partial}_{r_{1}}$, и коммутирование с $C_{r_{1}}$ суть $k$-линейные дифференцирования, для всех $r_{2} \geqslant 1$ получаем

$$
\begin{aligned}
\underline{\partial}_{r_{1}}\left(M^{r_{2}}\right)-\left[C_{r_{1}}, M^{r_{2}}\right] & =\sum_{r=0}^{r_{2}-1} M^{r}\left(\underline{\partial}_{r_{1}}(M)-\left[C_{r_{1}}, L\right]\right) M^{r_{2}-1-r}= \\
& =r_{2} \beta \xi_{m+r_{2}-1}+(\text { более низкий порядок } \xi) .
\end{aligned}
$$

В частности, степень оператора $\underline{\partial}_{r_{1}}\left(M^{r_{2}}\right)-\left[C_{r_{1}}, M^{r_{2}}\right]$ по $\xi$ не ограничена для $r_{2}$, стремящихся к бесконечности. Это противоречит предыдущему выводу о том, что эта степень ограничена $r_{1}-2$. Таким образом, наше предположение неверно, и, следовательно, все уравнения Лакса для $M$ должны выполняться. Это завершает доказательство теоремы. 
ЗАМЕЧАниЕ 3. Отношения нулевой кривизны (46) и (47) указывают на существование линейных систем, из которых формируются условия совместимости. Такие системы, которые будут представлены в следующем разделе, дают ключ к построению решений иерархий.

\section{7. ЛИНЕАРИЗАЦИЯ}

Нашей отправной точкой являются реализация $\left(\underline{R}, \underline{\partial},\left\{\underline{\partial}_{r}\right\}\right)$ строгой иерархии КП и реализация $\left(R, \partial,\left\{\partial_{i}\right\}\right)$ иерархии КП. Пусть $M$ - потенциальное решение первой иерархии в $\underline{R}\left[\xi, \xi^{-1}\right)$, а $L$ - потенциальное решение второй иерархии в $R\left[\xi, \xi^{-1}\right)$. Цель настоящего раздела - с одной стороны, описать линейную систему в подходящем $\underline{R}\left[\xi, \xi^{-1}\right)$-модуле, из которой будут следовать уравнения Лакса строгой иерархии КП и, с другой стороны, представить линейную систему в подходящем $R\left[\xi, \xi^{-1}\right)$-модуле, которая приводит к уравнениям Лакса иерархии КП. Для потенциального решения $M$ строгой иерархии КП должны выполняться равенства

$$
M \phi=z \phi, \quad \underline{\partial}_{r}(\phi)=C_{r}(\phi), \quad r \geqslant 1 .
$$

Эта система называется линеаризацией строгой иерархии КП. В случае иерархии КП потенциальное решение $L$ должно удовлетворять так называемой линеаризации иерархии КП, которая задается уравнениями

$$
L \psi=z \psi, \quad \partial_{i}(\psi)=B_{i}(\psi), \quad i \geqslant 1 .
$$

Прежде чем определить $\psi$ и $\phi$, мы покажем, какие манипуляции приводят к уравнениям Лакса для $M$ и $L$, а затем опишем условия, при которых они будут иметь место.

Применим дифференцирование $\underline{\partial}_{r}$ к первому из уравнений (48). Используя правило Лейбница для действия $\underline{\partial}_{r}$ на $M \phi$ и тот факт, что $z$ есть скаляр относительно и $\underline{R}[\xi]$-действия, и $\underline{\partial}_{r}$-действия, заменим второе уравнение и получим

$$
\begin{aligned}
\underline{\partial}_{r}(M \phi-z \phi) & =\underline{\partial}_{r}(M) \phi+M \underline{\partial}_{r}(\phi)-z \underline{\partial}_{r}(\phi)= \\
& =\underline{\partial}_{r}(M) \phi+M C_{r} \phi-C_{r}(z \phi)=\left(\underline{\partial}_{r}(M)-\left[C_{r}, M\right]\right) \phi=0 .
\end{aligned}
$$

Если мы можем отбросить функцию $\phi$, то $M$ будет удовлетворять уравнениям Лакса строгой иерархии КП.

Аналогично, применяя дифференцирование $\partial_{i}$ к первому из уравнений (49), приходим $\mathrm{K}$

$$
\begin{aligned}
\partial_{i}(L \psi-z \psi) & =\partial_{i}(L) \psi+L \partial_{i}(\psi)-z \partial_{i}(\psi)= \\
& =\partial_{i}(L) \psi+L B_{i} \psi-B_{i}(z \psi)=\left(\partial_{i}(L)-\left[B_{i}, L\right]\right) \psi=0 .
\end{aligned}
$$

Таким образом, если мы сможем не учитывать функцию $\psi$ в последнем уравнении, то получим уравнения Лакса для $L$.

Чтобы использовать уравнения (48), нам нужно левостороннее действие таких операторов, как $M$ и все $\left\{C_{r}\right\}$. Поэтому мы построим соответствующий $\underline{R}\left[\xi, \xi^{-1}\right)$-модуль, и вид его элементов будет определять решение (48) для тривиального решения $M=\xi$. В этом случае $C_{r}=\xi^{r}=M^{r}$ для всех $r \geqslant 1$, и уравнения (48) превращаются в

$$
M \phi=z \phi \quad \text { и } \quad \underline{\partial}_{r}(\phi)=M^{i}(\phi)=z^{r} \phi \quad \text { для всех } \quad r \geqslant 1 .
$$


В частности, можно заметить, что приближение первого порядка потока, соответствующего $\underline{\partial}_{r}$, есть умножение на $z^{r}$. Пусть $s_{r}$ определяет параметр для потока, соответствующего $\underline{\partial}_{r}$, так что $\underline{\partial}_{r}$ действует как взятие частной производной $\partial / \partial s_{r}$. Тогда уравнения линеаризации можно формально проинтегрировать. Итак, рассмотрим формальный ряд

$$
\phi_{0}=e^{\sum_{r=1}^{\infty} s_{r} z^{r}} .
$$

C введенной операцией $\underline{\partial}_{r}$ он удовлетворяет уравнениям линеаризации для тривиального решения строгой иерархии КП. Пространством, для которого можно использовать уравнения (48), является пространство $\mathcal{O}$ так называемых осциллирующих функций, которое можно понимать как множество возмущений тривиального решения $\phi_{0}$. Оно определяется следующим образом:

$$
\mathcal{O}=\left\{\left(\sum_{j=-\infty}^{N} a_{j} z^{j}\right) e^{\sum_{r=1}^{\infty} s_{r} z^{r}}=\left(\sum_{j=-\infty}^{N} a_{j} z^{j}\right) \phi_{0} \mid a_{j} \in R \text { для всех } j\right\} .
$$

Отметим, что произведение $\left(\sum_{j=-\infty}^{N} a_{j} z^{j}\right) \psi_{0}$ в элементах $\mathcal{O}$ формально. Если мы выразим $\psi_{0}$ через элементарные функции Шура, а именно

$$
e^{\sum_{i=1}^{\infty} s_{r} z^{r}}=\sum_{n=0}^{\infty} p_{n}(s) z^{n}
$$

то это произведение как ряд по степеням $z$ и $z^{-1}$ формально равно

$$
\left(\sum_{j=-\infty}^{N} a_{j} z^{j}\right) e^{\sum_{r=1}^{\infty} s_{r} z^{r}}=\sum_{\ell \in \mathbb{Z}}\left(\sum_{k=0}^{\infty} a_{\ell-k} p_{k}\right) z^{\ell}
$$

Поэтому, чтобы говорить о коэффициентах $\sum_{k=0}^{\infty} a_{\ell-k} p_{k}$ при всех $\ell \in \mathbb{Z}$, требуются соответствующие условия сходимости. Этого можно достичь, рассматривая произведение в соответствующем классе граничных значений (см. работу [3]). Естественное вложение $k\left[s_{r}\right]$ такое, как $k$-подалгебра $R$, а также тот факт, что $\underline{\partial}_{r}$ являются соответствующими продолжениями операции дифференцирования $\partial / \partial s_{r}$ на $k\left[s_{r}\right]$, помогают поместить эти коэффициенты в $R$. Пространство $\mathcal{O}$ становится $\underline{R}\left[\xi, \xi^{-1}\right)$-модулем согласно естественному продолжению действий

$$
\begin{aligned}
& b .\left(\sum_{j=-\infty}^{N} a_{j} z^{j}\right) \phi_{0}=\left(\sum_{j=-\infty}^{N} b a_{j} z^{j}\right) \phi_{0}, \quad b \in R \\
& \xi \cdot\left(\sum_{j=-\infty}^{N} a_{j} z^{j}\right) \phi_{0}=\left(\sum_{j=-\infty}^{N} \partial\left(a_{j}\right) z^{j}+\sum_{j=-\infty}^{N} a_{j} z^{j+1}\right) \phi_{0} .
\end{aligned}
$$

Мы также предположим, что каждый $\underline{\partial}_{r}$ действует на $\mathcal{O}$ согласно правилу Лейбница

$$
\underline{\partial}_{r}\left(\left(\sum_{j=-\infty}^{N} a_{j} z^{j}\right) \phi_{0}\right)=\left(\sum_{j=-\infty}^{N} \underline{\partial}_{r}\left(a_{j}\right) z^{j}+\sum_{j=-\infty}^{N} a_{j} z^{j+r}\right) \phi_{0} .
$$

Это определит действие всех операторов, которые присутствуют в уравнениях (48). Отметим, что $\mathcal{O}$ является свободным $\underline{R}\left[\xi, \xi^{-1}\right)$-модулем с образующим $\phi_{0}$, поскольку выполнено равенство

$$
\left(\sum_{j} p_{j} \xi^{j}\right) \phi_{0}=\left(\sum_{j} p_{j} z^{j}\right) \phi_{0}
$$


Таким образом, если мы имеем в $\mathcal{O}$ соотношения $Q \phi=0$, где $Q \in \underline{R}\left[\xi, \xi^{-1}\right)$, и $\phi=P \phi_{0}$, где $P \in \underline{R}\left[\xi, \xi^{-1}\right)$, то можно сделать вывод, что $Q P=0$ и, более того, $Q=0$, если $P$ обратим. И это случай следующего класса осциллирующих функций: элемент $\phi \in \mathcal{O}$ называется осщиллирующей функцией типа $\alpha_{\ell} z^{\ell}$, где $\alpha_{\ell}$ обратим в $\underline{R}$, если она представима в виде

$$
\phi=\phi(t, z)=\left\{\alpha_{\ell} z^{\ell}+\sum_{k<\ell} \alpha_{k} z^{k}\right\} \phi_{0}=K . \phi_{0}, \quad K=\sum_{k \leqslant \ell} \alpha_{k} \xi^{k} .
$$

Осциллирующая функция $\phi$ типа $\alpha_{\ell} z^{\ell}$ называется волновой функцией строгой иерархии КП, если существует оператор $M$ такой, что уравнения (48) выполнены для $M$ и $\phi$. Отметим, что в этом случае $M$ есть решение строгой иерархии КП, поскольку все преобразования, описанные в настоящем разделе, здесь правомерны. Тогда оператор $M$ полностью определяется функцией $\phi$. Если $\phi=K . \phi_{0}$, как в формуле (53), то первое из уравнений (48) может быть записано в виде

$$
M \phi=M K . \phi_{0}=z \phi=z K . \phi_{0}=K \xi . \phi_{0},
$$

и, значит, $M$ получается одеванием $\xi$ оператором $K$, т. е. $M=K \xi K^{-1}$. Отметим, что для получения уравнений (48) достаточно доказать более слабый результат.

УтВЕРЖДЕНИЕ 3. Пусть ф есть осииллирующая функиия типа $\alpha_{\ell} z^{\ell}$, а M оператор первого порядка в $\underline{R}\left[\xi, \xi^{-1}\right)$ вида (29). Предположим, что для любого $r \geqslant 1$ существует дифберенииальныи оператор $Q_{r} \in \underline{R}[\xi]$ без постоянного члена такой, что

$$
M \phi=z \phi, \quad \underline{\partial}_{r}(\phi)=Q_{r} \phi .
$$

Тогда для любого $r \geqslant 1$ выполнено $Q_{r}=C_{r}$. В частности, $M$ есть решение строгой иерархии КП.

ДоказАтельство. Пусть для $K$ имеет место равенство $\phi=K \phi_{0}$. Как было показано, первое уравнение гарантирует, что $M=K \xi K^{-1}$. По определению действия $\underline{\partial}_{r}$ на $\mathcal{O}$ имеем, с одной стороны,

$$
\underline{\partial}_{r}(\phi)=\left\{\underline{\partial}_{r}(K)+K \xi^{r}\right\} \cdot \phi_{0},
$$

а с другой стороны, это должно быть равно $Q_{r} K . \phi_{0}$. Отсюда получаем тождество

$$
\underline{\partial}_{r}(K) K^{-1}+K \xi^{r} K^{-1}=\underline{\partial}_{r}(K) K^{-1}+M^{r}=Q_{r} .
$$

Поскольку $\underline{\partial}_{r}(K) K^{-1}$ содержит только члены степени не выше нуля по $\xi$, то, рассматривая строго дифференциальную часть и с левой, и с правой стороны равенства, получим $M_{>0}^{r}=\left(Q_{r}\right)_{>0}=Q_{r}$. Утверждение доказано.

Предположим, что имеются две волновые функции $\phi_{1}=K_{1}$. $\phi_{0}$ и $\phi_{2}=K_{2} . \phi_{0}$ строгой иерархии КП, соответствующие одному и тому же решению $M$ строгой иерархии КП, т. е. имеют место равенства

$$
M \phi_{k}=z \phi_{k} \quad \text { и } \quad \underline{\partial}_{r}\left(\phi_{k}\right)=C_{r} \psi_{k}, \quad k=1,2 .
$$

Тогда, прежде всего, нам известно, что $K:=K_{1}^{-1} K_{2}$ коммутирует с $\xi$. Следовательно, коэффициенты $K$ постоянны для дифференцирования $\partial$, т. е. $\partial(K)=0$. Более того, из доказательства утверждения 3 следует, что для всех $r \geqslant 1$ выполнены

$$
\underline{\partial}_{r}\left(K_{1}\right)=\left(C_{r}-M^{r}\right) K_{1}, \quad \underline{\partial}_{r}\left(K_{2}\right)=\left(C_{r}-M^{r}\right) K_{2} .
$$


Это означает, что коэффициенты $K$ являются константами для всех дифференцирований $\underline{\partial}_{r}$, так как

$$
\begin{aligned}
\underline{\partial}_{r}(K) & =-K_{1}^{-1} \underline{\partial}_{r}\left(K_{1}\right) K_{1}^{-1} K_{2}+K_{1}^{-1} \underline{\partial}_{r}\left(K_{2}\right)= \\
& =-K_{1}^{-1}\left(C_{r}-M^{r}\right) K_{1} K_{1}^{-1} K_{2}+K_{1}^{-1} \underline{\partial}_{r}\left(K_{2}\right)= \\
& =K_{1}^{-1}\left(-\left(C_{r}-M^{r}\right) K_{2}+\underline{\partial}_{r}\left(K_{2}\right)\right)=0 .
\end{aligned}
$$

Таким образом, доказано следующее утверждение.

СЛЕДСТвИЕ 2. Если $\phi_{1}=K_{1} . \phi_{0} u \phi_{2}=K_{2} . \phi_{0}$ суть волновые функиии строгой иерархии $K \Pi$, соответствующие одному и тому же решению $M$, то элемент $K:=K_{1}^{-1} K_{2}$ в $D(0)$ постоянен для всех соответствующих потоков, т. е. $\underline{\partial}_{r}(K)=$ $\underline{\partial}(K)=0$ для всех $r \geqslant 1$.

В случае иерархии КП можно построить аналогичный $R\left[\xi, \xi^{-1}\right)$-модуль. Снова следует начать с линеаризации для тривиального решения $L_{0}=\xi$ этой иерархии:

$$
L_{0} \psi=z \psi, \quad \partial_{i}(\psi)=L_{0}^{i}(\psi)=z^{i} \psi, \quad i \geqslant 1 .
$$

Пусть $t_{i}$ - параметр для потока, соответствующего $\partial_{i}$, так что $\partial_{i}$ действует как взятие частной производной $\partial / \partial t_{i}$ по $t_{i}$. Тогда линеаризация имеет решение

$$
\psi_{0}=e^{\sum_{i=1}^{\infty} t_{i} z^{i}} .
$$

Соответствующий $R\left[\xi, \xi^{-1}\right)$-модуль снова есть множество $\mathcal{M}$ возмущений тривиального решения $\psi_{0}$, а именно

$$
\mathcal{M}=\left\{\left(\sum_{j=-\infty}^{N} a_{j} z^{j}\right) e^{\sum_{i=1}^{\infty} t_{i} z^{i}}=\left(\sum_{j=-\infty}^{N} a_{j} z^{j}\right) \psi_{0} \mid a_{j} \in R \text { для всех } j\right\},
$$

и элементы $\mathcal{M}$ также называются осииллирующими функииями. Пространство $\mathcal{M}$ можно сделать $R\left[\xi, \xi^{-1}\right)$-модулем способом, аналогичным структуре $\underline{R}\left[\xi, \xi^{-1}\right)$-модуля на $\mathcal{O}$. Более того, $\mathcal{M}$ будет свободным $R\left[\xi, \xi^{-1}\right)$-модулем с образующим $\psi_{0}$.

Мы снова выделим специальный класс элементов $\mathcal{M}$ : элемент $\psi \in \mathcal{M}$ называется осциллирующей функцией типа $z^{\ell}$, если он имеет вид

$$
\psi=\psi(t, z)=\left\{z^{\ell}+\sum_{k<\ell} \alpha_{k} z^{k}\right\} \psi_{0}=K . \psi_{0}, \quad K=\xi^{\ell}+\sum_{k<\ell} \alpha_{k} \xi^{k} .
$$

Осциллирующая функция $\psi$ типа $z^{\ell}$ называется волновой функцией иерархии КП, если существует оператор $L$ вида (27) такой, что уравнения (49) выполнены для $L$ и $\psi$. Отметим, что в этом случае $L$ есть решение иерархии КП, поскольку все преобразования, описанные в этом разделе, правомерны, и если $\psi=K . \psi_{0}$, то $L$ получается одеванием $\xi$ оператором $K$, т. е. $L=K \xi K^{-1}$. В постановке КП справедливы аналоги утверждения 3 и следствия 2. Мы сформулируем их для полноты изложения; доказательства повторяют приведенные выше.

УтВеРЖДЕНИЕ 4. 1. Пусть $\psi$ - осииллирующая функиия типа $z^{\ell}$ u L есть оператор первого порядка в $R\left[\xi, \xi^{-1}\right)$, который представляет собой потенииальное решение иерархии КП. Предположим, что для любого $i \geqslant 1$ существует дифференииальный оператор $P_{i} \in R[\xi]$ такой, что

$$
L \psi=z \psi, \quad \partial_{i}(\psi)=P_{i} \psi .
$$


Тогда для любого $i \geqslant 1$ выполнено $P_{i}=B_{i}$. В частности, $L$ есть решение иерархии КП.

2. Пусть $\psi_{1}=K_{1} . \psi_{0}$ и $\psi_{2}=K_{2} . \psi_{0}$ - волновые функции иерархии КП, соответствуюшие одному и тому же решению $L$. Тогда одевающие операторы $K_{1} u K_{2}$ отличаются только элементом $K$ в $D(0)_{1}$, который является постоянным для всех соответствующих дифференцирований, т.е. для всех $i \geqslant 1$

$$
\partial_{i}(K)=\partial(K)=0 .
$$

ЗАмЕчАниЕ 4. В работе [3] мы дадим геометрическую постановку, позволяющую строить волновые функции строгой иерархии КП, в которой произведение возмущений и экспоненциальный сомножитель сходятся.

\section{Список литературы}

[1] K. Ueno, K. Takasaki, "Toda lattice hierarchy", Group Representations and Systems of Differential Equations (Tokyo, December 20-27, 1982), Advanced Studies in Pure Mathematics, 4, North-Holland, Amsterdam-New York-Oxford, 1984, 1-95.

[2] G. Segal, G. Wilson, Inst. Hautes Études Sci. Publ. Math., 61 (1985), 5-65.

[3] G. F. Helminck, A. G. Helminck, E. A. Panasenko, Solutions of the Strict KP Hierarchy, in preparation.

[4] M. A. Olshanetsky, A. M. Perelomov, Phys. Rep., 71:5 (1981), 313-400.

[5] M. Guest, Harmonic Maps, Loop Groups and Integrable Systems, London Mathematical Society Student Texts, 38, Cambridge Univ. Press, Cambridge, 1997.

[6] B. Kostant, Adv. Math., 34:3 (1979), 195-338.

[7] P. D. Lax, Commun. Pure Appl. Math., 21:5 (1968), 467-490.

[8] M. Mulase, Adv. Math., 54:1 (1984), 57-66. 